\title{
BROWN REPRESENTABILITY FOR STABLE CATEGORIES
}

\author{
PETER JØRGENSEN
}

\begin{abstract}
This paper proves Brown Representability for stable categories. These categories are obtained from additive categories by declaring all morphisms which factor through objects from a fixed pre-enveloping class to be zero. Brown Representability for such categories is obtained by first proving that stable categories are what Brown in [2] calls "abstract homotopy categories", next using Brown's theory for these categories.

The result is applied to two cases: one is a category of complexes of modules over a ring. Here we recover a representability theorem for functors on derived categories, first given by Neeman in [11, Thm. 3.1]. The other case is the category of modules over an Artinian ring. Here we obtain a result on representability, Theorem 4.4 , which appears to be new.
\end{abstract}

\section{Introduction}

Brown Representability in various incarnations. Brown Representability is a central piece of wisdom to homotopy-theorists. So it is certainly something that one needs to mimick if one wants to mimick homotopy-theory successfully in a non-topological setting. That is the philosophy of this paper, which deals (roughly speaking) with homotopy-theory for modules, and culminates in a version of Brown Representability.

Let us take a more leisurely trip through some history and background for this paper. The first version of Brown Representability, proved in the fundamental paper [3], is a result in topology. It considers a functor $H$ defined on the homotopy-category of topological spaces, and gives an easy condition which is equivalent to the representability of $H$ (a functor is called representable if it is equivalent to a functor on the form $\operatorname{Hom}(-, X)$ ). Later versions of Brown's result do the same thing, but on other categories. One should think of these other categories as being analogous to the homotopycategory of topological spaces, and think of results such as the generalizations of Brown's result as being part of the "homotopical algebra" of these categories; cf. [12].

Received February 18, 1997. 
Notable among the abstract versions of Brown Representability is Brown's own result [2, Thm. 2.8], which works for any category $\mathscr{C}$ satisfying a list of axioms which resemble topological facts (Brown calls such a $\mathscr{C}$ "an abstract homotopy-category"). One should also mention Neeman's version [11, Thm. 3.1], which works on many triangulated categories.

The present paper also gives an abstract Brown-theorem, carried by the so-called stable categories from relative homological algebra (see [1] or below for their definition). These categories were introduced into homological algebra by Hilton in [7, chp. 13], as a vehicle for transferring homotopytheoretical ideas into homological algebra. They are, in fact, direct additive analogues of the usual homotopy-category of topological spaces, that is, of the category on which Brown's original result lives (we shall expound on the analogy in section 1 below). It is thus obvious to seek a result of Brown-type for stable categories.

The full story of the paper is a bit longer than so far indicated. The modus operandi below is not to aim directly for a Brown-result; instead, we will use Brown's own abstract theory from [2] as a stepping-stone. Our main result, Theorem 2.1, states that under suitable conditions, a stable category is one of the "abstract homotopy-categories" defined in [2]; as mentioned, this means that it satisfies a certain list of axioms resembling facts known from topology, see [2, sec. 2]. By the results from [2], this gives us Brown Representability for stable categories. (But not without one final hurdle: not all abstract homotopy-categories support good versions of Brown's result. This is why theorem 2.1, and not Brown Representability itself, is our main result.)

Along the way, we will have occasion to use the concept of right-triangulated categories introduced in [1] a great deal, since stable categories are right-triangulated. Indeed, many of the nice properties of stable categories are encoded in the axioms of right-triangulated categories - so many, that I think of right-triangulated categories in general, and not just of stable categories, as being additive cousins of the homotopy-category of topological spaces.

Applications. The developments described have various applications, of which two are carried through below. One, described in section 3 , is really just an example, which shows how an instance of Neeman's result [11, Thm. 3.1], dealing with cohomological functors on the derived category of a ring, is contained in the present theory: if $A$ is a ring and $H: D(A) \longrightarrow \mathbf{A b}$ a contravariant additive functor to the category of abelian groups, then $H$ is representable if and only if $H$ is cohomological and sends small coproducts to the corresponding products. The other application, described in section 4 , 
shows how the present theory can be used on additive functors defined on a stable module-category over an Artinian ring. We give representability-criteria for such functors in Theorem 4.4. This application ties in with the fact that stable categories are one of the central tools in the modern theory of Artinian rings, see [5]. We also widen the scope, and contemplate what a "cohomology-theory" defined on the stable module-category might look like. It turns out that when we borrow the topologists' definition, such a theory can be represented by what we will call an $\Omega$-spectrum of modules; we also borrow this definition from topology. That this should be so is no surprise: in the topological case, it is a classical result contained in [3].

A few words about an obvious missing application: the pre-enveloping classes appearing in the theory below are really a device from relative homological algebra. It therefore seems likely that applications should exist in that area, although I have yet to find some.

Layout of the paper. To sum up, section 1 below is preparatory, and shows how some constructions from topological homotopy-theory can be transferred to stable categories. The idea is that, since Brown's abstract homotopy-categories are characterized by axioms reminiscent of topological homotopy-theory, our section 1 will help us to connect stable categories with Brown's work. Section 2 contains the central proof that stable categories are "abstract homotopy-categories" in the terminology from [2]; this implies by [2, Thm. 2.8] that stable categories admit a Brown Representability theorem. Section 3 applies the theory to the case of complexes of modules, reproving a special case of the result [11, Thm. 3.1], and section 4 applies the theory to modules over Artinian rings.

Comments about stable categories. One thing to note is that these categories have been named in a most unfortunate way, since their position close to homotopy-theory makes one believe that the word "stable" in "stable category" must have something to do with stable homotopy. This is not the case! In fact, as mentioned, I think of stable categories as additive analogues of the usual homotopy-category of topological spaces, i.e. of the unstable homotopy-category.

Another comment concerns abstract right-triangulated categories. As indicated above, I think of all these categories, not just of stable categories, as additive generalizations of the homotopy-category of topological spaces. And so of course I tried first to prove Brown Representability for an arbitrary right-triangulated category, not just for a stable category. However, this endeavour failed, because it turned out to be unclear how to perform a key construction, that of homotopy colimits, in the abstract case. In stable 
categories, however, the construction is not too difficult, and this is the reason why this paper deals only with stable categories.

Notation and mathematical background. Let $\mathbb{C}$ be a category, $\mathrm{X}$ a class of objects of $\mathfrak{C}$. We will follow the terminology from [4] and call a morphism $f: C_{1} \longrightarrow C_{2}$ an X-monic if

for $X \in \mathrm{X}$ and $g_{1}: C_{1} \longrightarrow X$, we can find $g_{2}: C_{2} \longrightarrow X$ with $g_{2} f=g_{1}$.

An X-monic $f: C \longrightarrow X$, where $X$ is in $\mathrm{X}$, is called an X-pre-envelope. We call $\mathrm{X}$ a pre-enveloping class if every object $C$ of $\mathbb{C}$ has an $\mathrm{X}$-pre-envelope.

As an example, think of $\mathfrak{C}$ as a category of modules, $X$ as the injective modules. Then the $X$-monics are the usual injective homomorphisms, and an $X$-pre-envelope is an injection into an injective module.

The notions dual to $\mathrm{X}$-monic and $\mathrm{X}$-pre-envelope are called $\mathrm{X}$-epic and $\mathrm{X}$ pre-cover; there is an ensuing notion of pre-covering class.

We use MacLane's definition of additive categories, see [9, sec. VIII.2]. If $\mathfrak{A}$ is an additive category with a pre-enveloping class $X$, we will call a morphism null-homotopic (with respect to $X$ ) if it factors through an object from $X$. It is easy to see that a morphism going out of the object $C$ is nullhomotopic precisely if it factors through any one of $C$ 's X-pre-envelopes. We call two different morphisms homotopic to each other (with respect to $X$ ) if their difference is null-homotopic. We construct the so-called stable category $\mathfrak{U}_{\mathrm{X}}$ by dividing away from $\mathfrak{A}$ the ideal of null-homotopic morphisms (so $\mathfrak{A}_{\mathrm{X}}$ has the same objects as $\mathfrak{A}$, but its morphisms are equivalence classes of morphisms from $\mathfrak{A})$. The canonical functor exhibiting $\mathfrak{A}_{\mathrm{X}}$ as factor of $\mathfrak{A}$ will be denoted $Q: \mathfrak{A} \longrightarrow \mathfrak{A}_{\mathrm{x}}$, and for a morphism $f$ in $\mathfrak{A}$, we follow [1] and write

$$
Q(f)=\underline{f} .
$$

Occasionally, we shall follow [7, chp. 13] and denote the homomorphismfunctor $\operatorname{Hom}_{\mathfrak{G}_{\mathrm{x}}}$ by $\pi$.

In [1], the notion of right-triangulated category was introduced, and it was realized that if one equips $\mathfrak{A}_{X}$ with a suitable suspension-functor $\Sigma$, and a suitable class of distinguished right-triangles $\Delta$, then $\left(\mathfrak{U}_{\mathrm{X}}, \Sigma, \Delta\right)$ is a righttriangulated category (to be more accurate, [1] really deals with the case where $\mathrm{X}$ is pre-covering and $\mathfrak{A}_{\mathrm{X}}$ left-triangulated, but everything can be dualized).

Finally, we will be using the notion of small sets a great deal. For an explanation of small sets and their significance to category theory, see $[9$, sec. I.6]. As shorthand, when we mean e.g. the coproduct of a family indexed by a small set, we talk of "a small coproduct". 


\section{Homotopical algebra in a stable category}

In this section, we shall see how some elementary constructions from topological homotopy-theory can be transferred to categories of the form $\mathfrak{A}_{\mathrm{x}}$ : homotopy-extension, factorization through the mapping-cylinder, some homotopy colimits, coproducts, and exact homotopy-sequences. They are all easy to perform in our additive setting. This section is very close in spirit to [7, chp. 13]; just like Hilton, we shall try to write all results up in a way making the formal resemblance to topological homotopy-theory as good as possible.

We begin with two "classical" results.

Lemma 1.1 (Homotopy-extension). Let $\mathfrak{A}$ be an additive category with a pre-enveloping class $\mathrm{X}$. Suppose that we have a diagram

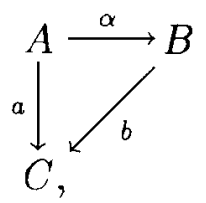

which is commutative up to $\mathrm{X}$-homotopy, that is, ba is homotopic to a, and in which $\alpha$ is $\mathrm{X}$-monic.

Then there exists a morphism $b^{\prime}: B \longrightarrow C$ such that

- $b^{\prime} \alpha=a$,

- $b^{\prime}$ is homotopic to $b$.

Proof. This is [7, Thm. 13.6]. Note that Hilton operates with $\mathrm{X}=\mathscr{I} n j$, the class of injectives, but that his proof works for a general pre-enveloping class $\mathrm{X}$.

Lemma 1.2 (Factorization through the mapping-cylinder). Let $\mathfrak{A}$ be an additive category with a pre-enveloping class $\mathrm{X}$. Any morphism $\alpha: A \longrightarrow B$ in $\mathfrak{A}$ can be factored as

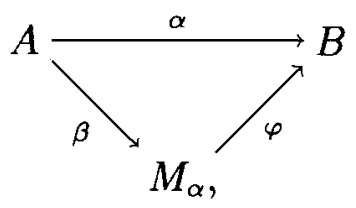

where

- $\beta$ is an X-monic,

- $\varphi$ is an X-homotopy-equivalence, i.e., $\underline{\varphi}$ is an isomorphism in $\mathfrak{H}_{\mathrm{X}}$.

Proof. This is also contained in [7], on page 132: Hilton operates with 
$\mathrm{X}=\mathscr{I} n j$, but his method works for general X's. Note that $M_{\alpha}$ is not in general uniquely determined by $\alpha$, so the notation $M_{\alpha}$ is a bit misleading.

We want to go on and study homotopy colimits in $\mathfrak{A}_{X}$. The following lemma paves the way.

Lemma 1.3. Let $\mathfrak{A}$ be an additive category with a pre-enveloping class $\mathrm{X}$. If we have a system in $\mathfrak{A}$,

$$
A_{1} \stackrel{\alpha_{1}}{\longrightarrow} A_{2} \stackrel{\alpha_{2}}{\longrightarrow} \cdots,
$$

then we can find a new system,

$$
B_{1} \stackrel{\beta_{1}}{\longrightarrow} B_{2} \stackrel{\beta_{2}}{\longrightarrow} \cdots,
$$

and morphisms $\varphi_{i}: B_{i} \longrightarrow A_{i}$, such that

- the diagram of A's and B's is commutative, that is, for each $i$ we have $\alpha_{i} \varphi_{i}=\varphi_{i+1} \beta_{i}$,

- each $\varphi_{i}$ is an $\mathrm{X}$-homotopy-equivalence,

- each $\beta_{i}$ is an X-monic.

Note that by the two first points, the B-system is isomorphic to the A-system in $\mathfrak{A}_{\mathrm{X}}$.

Proof. This simply consists in successive applications of Lemma 1.2:

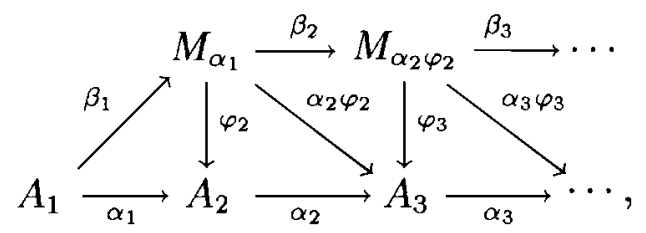

setting $B_{2}=M_{\alpha_{1}}$, and $B_{i}=M_{\alpha_{i-1} \varphi_{i-1}}$ for all $i \geq 3$.

We finish the diagram by setting $B_{1}=A_{1}$ and $\varphi_{1}=1$.

The lemma will be used for the following construction: suppose that we are given an additive category $\mathfrak{A}$ with a pre-enveloping class $\mathrm{X}$, and suppose that we have a system in $\mathfrak{H}_{\mathrm{X}}$ given by

$$
X_{1} \stackrel{f_{1}}{\longrightarrow} X_{2} \stackrel{f_{2}}{\longrightarrow} \cdots .
$$

We perform the construction from Lemma 1.3 to get a commutative diagram 


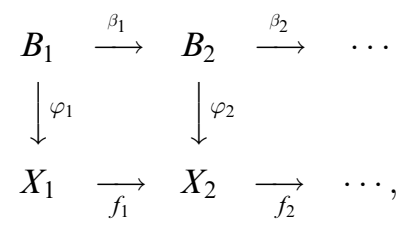

in which all the $\beta_{i}$ 's are $\mathrm{X}$-monics, and all the $\varphi_{i}$ 's are $\mathrm{X}$-homotopy-equivalences.

Now suppose that $\mathfrak{A}$ contains the colimit of the system $B_{1} \stackrel{\beta_{1}}{\longrightarrow} B_{2} \stackrel{\beta_{2}}{\longrightarrow} \cdots$. Write $X$ for this colimit, and let $b_{i}: B_{i} \longrightarrow X$ be the canonical maps. Set $g_{i}=\underline{b}_{i} \circ\left(\varphi_{i}\right)^{-1}$ (remember that $\varphi_{i}$ is a homotopyequivalence, so $\varphi_{i}$ is an invertible morphism in $\mathfrak{A}_{X}$ ). We then get morphisms $\underline{g_{i}}: X_{i} \longrightarrow X$ in $\overline{\mathfrak{A}}_{\mathrm{X}}$, and they are compatible with the $f_{i}$ 's.

Definition 1.4. We shall call an $X$ as constructed above a homotopy colimit of the system (1), and write

$$
X=\operatorname{hocolim} X_{i} .
$$

The $g_{i}$ 's will be called canonical maps (associated to the homotopy colimit of the system (1)).

But note that it is not obvious that our construction provides an $X$ which is unique.

The reason for introducing homotopy colimits is that they have some nice properties, well-known to topologists. They are expressed in the following two lemmas, cf. [2, Axiom (2.4)]:

LEMMA 1.5. Let $\mathfrak{2}$ be an additive category with a pre-enveloping class $\mathrm{X}$, and let the system (1) be given. Assume that an $X=$ hocolim $X_{i}$ as in Definition 1.4 can be constructed.

Then for any object $Z \in \mathfrak{U}$, the maps

$$
\operatorname{Hom}_{\mathfrak{I}_{\mathrm{x}}}\left(\underline{g_{i}}, Z\right): \operatorname{Hom}_{\mathfrak{I}_{\mathrm{x}}}(X, Z) \longrightarrow \operatorname{Hom}_{\mathfrak{A}_{\mathrm{x}}}\left(X_{i}, Z\right)
$$

induce a map

$$
\gamma: \operatorname{Hom}_{\mathfrak{G}_{\mathrm{x}}}(X, Z) \longrightarrow \lim \operatorname{Hom}_{\mathfrak{H}_{\mathrm{X}}}\left(X_{i}, Z\right),
$$

and $\gamma$ is an epimorphism.

Proof. In the category $\mathfrak{U}_{\mathrm{X}}$, the system of $X_{i}$ 's and the system of $B_{i}$ 's are isomorphic. So to prove that $\gamma$ is surjective, we can replace $X_{i}$ by $B_{i}$ and $g_{i}$ by $b_{i}$.

Let us suppose that we have an element in the limit

$$
\lim \operatorname{Hom}_{\mathfrak{A}_{\mathrm{x}}}\left(B_{i}, Z\right) \text {. }
$$


It will be given by a compatible family in $\mathfrak{A}_{\mathrm{x}}$,

$$
\left(\underline{h_{i}}: B_{i} \longrightarrow Z\right)_{i} \text {, }
$$

where the compatibility means that $\underline{h_{i+1}} \circ \beta_{i}=\underline{h_{i}}$ for every $i$.

We will now use Lemma 1.1 successively, to find morphisms $q_{i}$ which "improve" the $h_{i}$ 's. This can be done since all the $\beta_{i}$ 's are X-monics:

$1^{\circ}$ : Put $q_{1}=h_{1}$.

$2^{\circ}$ : Since $h_{2} \circ \beta_{1}$ is homotopic to $h_{1}$, it is also homotopic to $q_{1}$. So by Lemma 1.1 , we may choose $q_{2}: B_{2} \longrightarrow Z$ such that $q_{2} \circ \beta_{1}=q_{1}$, and such that $q_{2}$ is homotopic to $h_{2}$.

$3^{\circ}$ : Since $h_{3} \circ \beta_{2}$ is homotopic to $h_{2}$, it is also homotopic to $q_{2}$. So we may choose $q_{3}: B_{3} \longrightarrow Z$ such that $q_{3} \circ \beta_{2}=q_{2}$, and such that $q_{3}$ is homotopic to $h_{3}$.

If we go on in this way, we get a system

$$
\left(q_{i}: B_{i} \longrightarrow Z\right)_{i}
$$

which is compatible in $\mathfrak{A}$. But this induces $q$ : colim $B_{i} \longrightarrow Z$, that is, $q: X \longrightarrow Z$. And it is clear that if we view $\gamma(q)$ as a compatible system, its $i$ 'th component is $q_{i}$ which is equal to $\underline{h_{i}}$, so $\gamma(\underline{q})$ is equal to the lim-element given by the system (2).

This proves that an arbitrary element of $\lim \operatorname{Hom}_{\mathfrak{A}_{\mathrm{x}}}\left(B_{i}, Z\right)$ lies in the image of $\gamma$.

Lemma 1.6. Let $\mathfrak{O}$ be an additive category with a pre-enveloping class $\mathrm{X}$, and let the system (1) be given. Assume that an $X=$ hocolim $X_{i}$ as in Definition 1.4 can be constructed.

Then for any object $Z \in \mathfrak{A}$, the maps

$$
\operatorname{Hom}_{\mathfrak{U}_{x}}\left(Z, \underline{g_{i}}\right): \operatorname{Hom}_{\mathfrak{U}_{x}}\left(Z, X_{i}\right) \longrightarrow \operatorname{Hom}_{\mathfrak{U}_{\mathrm{x}}}(Z, X)
$$

induce a map

$$
\delta: \operatorname{colim} \operatorname{Hom}_{\mathfrak{I}_{X}}\left(Z, X_{i}\right) \longrightarrow \operatorname{Hom}_{\mathfrak{I}_{\mathbf{x}}}(Z, X) .
$$

Suppose now that $Z$ has the $\mathrm{X}$-pre-envelope $z: Z \longrightarrow Y$, and that both functors $\operatorname{Hom}_{\mathfrak{I}}(Z,-)$ and $\operatorname{Hom}_{\mathfrak{I}}(Y,-)$ preserve colimits of systems of the form

$$
A_{1} \stackrel{\alpha_{1}}{\longrightarrow} A_{2} \stackrel{\alpha_{2}}{\longrightarrow} \cdots
$$

where each $\alpha_{i}$ is an $\mathrm{X}$-monic. Then $\delta$ is an isomorphism.

Proof. Again we use that in the category $\mathfrak{A}_{\mathrm{X}}$, the system of $X_{i}$ 's and the system of $B_{i}$ 's are isomorphic: we replace $X_{i}$ by $B_{i}$ and $g_{i}$ by $b_{i}$.

But 


$$
\delta: \operatorname{colim} \operatorname{Hom}_{\mathfrak{I}_{\mathrm{X}}}\left(Z, B_{i}\right) \longrightarrow \operatorname{Hom}_{\mathfrak{H}_{\mathrm{X}}}(Z, X)
$$

can be computed to be

$$
\operatorname{colim} \frac{\operatorname{Hom}_{\mathfrak{I}}\left(Z, B_{i}\right)}{z^{*} \operatorname{Hom}_{\mathfrak{I}}\left(Y, B_{i}\right)} \longrightarrow \frac{\operatorname{Hom}_{\mathfrak{U}}(Z, X)}{z^{*} \operatorname{Hom}_{\mathfrak{I}}(Y, X)} .
$$

colim is exact, so we can "move it in" to numerator and denominator on the left-hand side. And both $\operatorname{Hom}_{\mathfrak{I}}(Z,-)$ and $\operatorname{Hom}_{\mathfrak{I}}(Y,-)$ preserve colimits of systems like the $B_{i}$-system. And $\operatorname{colim} B_{i}=X$, so we see that $\delta$ is an isomorphism.

It is convenient that $\mathfrak{A}_{\mathrm{X}}$ frequently has small coproducts.

Lemma 1.7. Let $\mathfrak{U}$ be an additive category with a pre-enveloping class $\mathrm{X}$. Suppose that $\mathfrak{I}$ has small coproducts, and that $\mathrm{X}$ is closed under such small coproducts.

Then the canonical functor $Q: \mathfrak{A} \longrightarrow \mathfrak{A}_{\mathrm{X}}$ preserves small coproducts. In particular, the category $\mathfrak{H}_{\mathrm{X}}$ has small coproducts.

ProOF. We let $\left\{A_{k}\right\}_{k \in K}$ be a small family in $\mathfrak{A}$, and denote by $i_{k}: A_{k} \longrightarrow \coprod_{\ell} A_{\ell}$ the corresponding injections. Choose an X-pre-envelope $\alpha_{k}: A_{k} \longrightarrow X_{k}$ for each $k \in K$. Note that $\coprod_{\ell} \alpha_{\ell}: \coprod_{\ell} A_{\ell} \longrightarrow \bigsqcup_{\ell} X_{\ell}$ is an Xpre-envelope.

We have $Q\left(i_{k}\right): Q\left(A_{k}\right) \longrightarrow Q\left(\amalg_{\ell} A_{\ell}\right)$, and we look at

$$
\begin{array}{ccc}
\operatorname{Hom}_{\mathfrak{I}_{\mathrm{x}}}\left(Q\left(\coprod_{\ell} A_{\ell}\right), Q(B)\right) & \longrightarrow & \prod_{k} \operatorname{Hom}_{\mathfrak{I}_{\mathrm{x}}}\left(Q\left(A_{k}\right), Q(B)\right) \\
\frac{\operatorname{Hom}_{\mathfrak{I}}\left(\coprod_{\ell} A_{\ell}, B\right)}{\left(\coprod_{\ell} \alpha_{\ell}\right)^{*} \operatorname{Hom}_{\mathfrak{I}}\left(\coprod_{\ell} X_{\ell}, B\right)} \longrightarrow & \prod_{k} \frac{\operatorname{Hom}_{\mathfrak{I}}\left(A_{k}, B\right)}{\alpha_{k}^{*} \operatorname{Hom}_{\mathfrak{I}}\left(X_{k}, B\right)},
\end{array}
$$

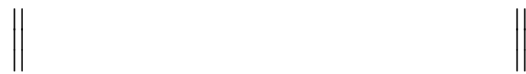

where the upper arrow is induced by the $Q\left(i_{k}\right)$, while the lower arrow is induced by the $i_{k}$. Since the lower arrow is easily seen to be an isomorphism, the upper arrow is also an isomorphism, and we conclude that $Q\left(\amalg_{\ell} A_{\ell}\right)$ is the coproduct of the $Q\left(A_{k}\right)$ 's, and that the canonical injections are the morphisms $Q\left(i_{k}\right): Q\left(A_{k}\right) \longrightarrow Q\left(\coprod_{\ell} A_{\ell}\right)$.

Finally, we look at exact homotopy-sequences. We will see that they exist for any right-triangulated category; in particular, they exist for $\mathfrak{U}_{X}$ since this category is right-triangulated by the arguments in [1].

Definition 1.8. Let $(\mathfrak{C}, \Sigma, \Delta)$ be a right-triangulated category; see [1, Def. $2.2]$ for the definition (or rather, the dual definition, namely that of left-triangulated categories).

A contravariant additive functor $H: \mathfrak{C} \longrightarrow \mathbf{A b}$ will be called half-exact if it satisfies: when 


$$
A \stackrel{a}{\longrightarrow} B \stackrel{b}{\longrightarrow} C \stackrel{c}{\longrightarrow} \Sigma(A)
$$

is in the class $\Delta$ of distinguished right-triangles, the sequence

$$
H(C) \stackrel{H(b)}{\longrightarrow} H(B) \stackrel{H(a)}{\longrightarrow} H(A)
$$

is exact.

Recall from [1] that a right-triangulated category is an additive category $\mathbb{C}$ with an additive endofunctor $\Sigma$ and a class of distinguished triangles $\Delta$, subject to Axioms (RT1) to (RT4). For instance, Axiom (RT2) for right-triangulated categories, dual to (LT2) in [1, Def. 2.2], states that if we have the distinguished right-triangle (3), then the right-triangle

$$
B \stackrel{b}{\longrightarrow} C \stackrel{c}{\longrightarrow} \Sigma(A) \stackrel{-\Sigma(a)}{\longrightarrow} \Sigma(B)
$$

is also distinguished. This makes it plain that if $H$ is half-exact, the distinguished right-triangle (3) gives rise to a long-exact sequence

$$
\begin{aligned}
& \cdots H(\Sigma(C)) \stackrel{H(\Sigma(b))}{\longrightarrow} H(\Sigma(B)) \stackrel{H(\Sigma(a))}{\longrightarrow} H(\Sigma(A)) \\
& \swarrow H(c) \\
& H(C) \underset{H(b)}{\longrightarrow} H(B) \underset{H(a)}{\longrightarrow} H(A) .
\end{aligned}
$$

LEMMA 1.9 (Valid for abstract right-triangulated categories). Let ( $\mathfrak{C}, \Sigma, \Delta)$ be a right-triangulated category. For any object $X \in \mathfrak{C}$, the functor $\operatorname{Hom}_{\mathfrak{C}}(-, X)$ is half-exact.

Proof. One can use the standard-proof from the theory of triangulated categories.

Note, however, that the same trick does not work in the second variable: the functor $\operatorname{Hom}_{\mathfrak{C}}(X,-)$ does not in general send right-triangles to exact sequences. The reason that the usual argument from triangulated categories does not work here is that $\Sigma^{-1}$ is not known to exist.

\section{Stable categories as abstract homotopy categories}

In this section, we shall prove our main result, Theorem 2.1: that categories of the form $\mathfrak{A}_{X}$ are what Brown in [2] calls "abstract homotopy-categories", when $\mathfrak{A}$ and $X$ have certain properties. As stated in the introduction, this is good because in many cases, it implies that $\mathfrak{U}_{\mathrm{X}}$ admits a Brown Representability Theorem which gives an easy necessary and sufficient criterion for an additive functor $F: \mathfrak{A}_{\mathbf{X}} \longrightarrow \mathbf{A b}$ to be representable ( $\mathbf{A b}$ is the category of abelian groups).

The reason that our main result is Theorem 2.1 instead of Brown Re- 
presentability itself is the "in many cases" above - even when we are in a case where Theorem 2.1 is valid, Brown Representability is not necessarily true.

Abstract homotopy-categories are simply categories satisfying some axioms resembling familiar facts from topological homotopy-theory (existence of homotopy colimits being the most important one). So the proof of Theorem 2.1 will simply consist in checking these axioms for a stable category. The proof is made possible by the results of section 1 which make manifest the homotopical nature of $\mathfrak{A}_{\mathrm{X}}$.

It will be handy to introduce short-hand notation for some conditions which can be imposed on an additive category $\mathfrak{A}$ with a pre-enveloping class $\mathrm{X}$ :

- Condition (A): any X-monic has a cokernel.

- Condition (B): $\mathfrak{A}$ has small coproducts, that is, coproducts of families indexed by small sets.

- Condition (C): $\mathrm{X}$ is closed with respect to small coproducts (we only impose this condition when it makes sense, i.e. when condition (B) is also in force).

- Condition (D): a system of the form

$$
A_{1} \stackrel{\alpha_{1}}{\longrightarrow} A_{2} \stackrel{\alpha_{2}}{\longrightarrow} \cdots,
$$

where each $\alpha_{i}$ is an $\mathbf{X}$-monic, has a colimit in $\mathfrak{A}$.

Note that in practice, the conditions are frequently satisfied. For instance, when $\mathfrak{A}$ is abelian and closed under small colimits, conditions (A), (B) and (D) hold automatically.

Now we can prove the main result:

THeOREM 2.1. Let $\mathfrak{A}$ be an additive category with a pre-enveloping class $\mathrm{X}$, satisfying conditions (A) to (D).

Write $\mathscr{C}=\mathfrak{H}_{\mathrm{X}}$, and suppose that we have chosen a small set $\mathscr{C}_{0}$ of objects in $\mathfrak{I}$; we will think of this small set as a full subcategory of $\mathscr{C}$. Assume

$\left(1^{\circ}\right)$ : if $C_{1}, \ldots, C_{N}$ are elements of $\mathscr{C}_{0}$, then $\mathscr{C}_{0}$ contains a coproduct of $C_{1}, \ldots, C_{N}$.

$\left(2^{\circ}\right)$ : if $C \in \mathscr{C}_{0}$, then $\mathrm{Hom}_{\mathfrak{H}}(C,-)$ respects the colimits mentioned in condition $(D)$.

$\left(3^{\circ}\right):$ if $C \in \mathscr{C}_{0}$, then we can find an $\mathrm{X}$-pre-envelope $C \longrightarrow X$ where also $X \in \mathscr{C}_{0}$.

$\left(4^{\circ}\right)$ : if we have an X-monic $\gamma: C_{1} \longrightarrow C_{2}$ with $C_{1}$ and $C_{2}$ in $\mathscr{C}_{0}$, then the cokernel of $\gamma$ can be chosen in $\mathscr{C}_{0}$.

Then the pair $\left(\mathscr{C}, \mathscr{C}_{0}\right)$ is an abstract homotopy-category, in the sense of $[2, \mathrm{p}$. 79]. 
Proof. Before starting the actual proof, let us briefly review Brown's axioms for abstract homotopy-categories, to get a feeling for what we are trying to prove. Brown considers a pair $\left(\mathscr{C}, \mathscr{C}_{0}\right)$ where $\mathscr{C}_{0}$ is a subcategory of $\mathscr{C}$. His first two axioms, [2, (2.1) and (2.2)], are "background": they just require that $\mathscr{C}_{0}$ is a small full subcategory of $\mathscr{C}$, that $\mathscr{C}$ has small coproducts, and that $\mathscr{C}_{0}$ is closed under finite coproducts.

Axiom [2, (2.3)] requires the existence of what one can call homotopy push-outs (though Brown calls them "equalizers"): for $f_{i}: A \longrightarrow X_{i}$ with $i=1,2$, one must be able to form $g_{i}: X_{i} \longrightarrow Z$ such that $g_{1} f_{1}=g_{2} f_{2}$ and such that: if $g_{i}^{\prime}: X_{i} \longrightarrow Z^{\prime}$ satisfy $g_{1}^{\prime} f_{1}=g_{2}^{\prime} f_{2}$, we can find $h: Z \longrightarrow Z^{\prime}$ with $g_{i}^{\prime} h=g_{i}$. Note that $h$ is not assumed to be unique. It is also required that if $A$ and the $X_{i}$ 's are in $\mathscr{C}_{0}$, then $Z$ can be chosen in $\mathscr{C}_{0}$.

Finally, Axiom [2, (2.4)] requires the existence of homotopy colimits of sequences: for a sequence $X_{1} \longrightarrow X_{2} \longrightarrow \cdots$, there should exist an $X$ and compatible maps $g_{i}: X_{i} \longrightarrow X$ such that the following two conditions hold:

[2, (2.4)(i)]: if $Z \in \mathscr{C}_{0}$, then $\operatorname{colim}\left(g_{n *}\right): \operatorname{colim} \operatorname{Hom}_{\mathscr{C}}\left(Z, X_{n}\right) \stackrel{\cong}{\longrightarrow} \operatorname{Hom}_{\mathscr{C}}(Z, X)$ is a bijection.

[2, (2.4)(ii)]: if $Z \in \mathscr{C}$, then $\left.\lim \left(g_{n}^{*}\right): \operatorname{Hom}_{\mathscr{C}}(X, Z) \longrightarrow \lim _{\mathscr{C}} \operatorname{Hom}_{\mathscr{C}}\left(X_{n}, Z\right)\right)$ is a surjection.

We shall now check these axioms for the case at hand.

Axiom [2, (2.1)]: requires $\mathscr{C}_{0}$ to be small and full, which it is.

Axiom [2, (2.2)]: small coproducts in $\mathscr{C}$ exist because of Lemma 1.7. And $\mathscr{C}_{0}$ has finite coproducts because of condition $\left(1^{\circ}\right)$.

Axiom [2, (2.3)]: suppose that we are given morphisms

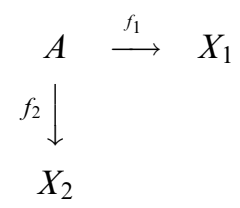

in $\mathscr{C}$. Using the right-triangulated structure of $\mathscr{C}=\mathfrak{A}_{\mathrm{X}}$, we can construct a distinguished right-triangle

$$
A \stackrel{\left(\begin{array}{c}
f_{1} \\
-f_{2}
\end{array}\right)}{\longrightarrow} X_{1} \coprod X_{2} \stackrel{\left(g_{1}, g_{2}\right)}{\longrightarrow} Z \longrightarrow \Sigma(A) .
$$

We claim that the $Z$ thus constructed satisfies Brown's axiom: let $g_{i}^{\prime}: X_{i} \longrightarrow Z^{\prime}$ be given such that $g_{1}^{\prime} f_{1}=g_{2}^{\prime} f_{2}$. We combine the $g_{i}^{\prime \prime}$ s to $\left(g_{1}^{\prime}, g_{2}^{\prime}\right): X_{1} \amalg X_{2} \longrightarrow Z^{\prime}$, which then satisfies $\left(g_{1}^{\prime}, g_{2}^{\prime}\right) \circ\left(\begin{array}{c}f_{1} \\ -f_{2}\end{array}\right)=0$. By 
Lemma 1.9 , we can find $h: Z \longrightarrow Z^{\prime}$ such that $h \circ\left(g_{1}, g_{2}\right)=\left(g_{1}^{\prime}, g_{2}^{\prime}\right)$, but then, $h g_{i}=g_{i}^{\prime}$ for $i=1,2$.

We also need to see that if $A$ and the $X_{i}$ 's are in $\mathscr{C}_{0}$, then we can choose $Z$ in $\mathscr{C}_{0}$. But if $A \stackrel{a}{\longrightarrow} Y$ is an $\mathrm{X}$-pre-envelope then by the procedure dual to that in [1, p. 5026], we form $Z$ as the push-out

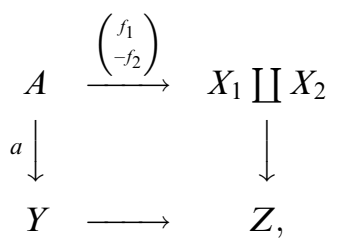

that is, we form $Z$ as the cokernel of the $\mathrm{X}$-monic

$$
A \stackrel{\left(\begin{array}{c}
-a \\
f_{1} \\
-f_{2}
\end{array}\right)}{\longrightarrow} Y \coprod X_{1} \coprod X_{2} .
$$

By condition $\left(3^{\circ}\right)$, we may choose $Y$ in $\mathscr{C}_{0}$. But then by condition $\left(4^{\circ}\right)$, we can suppose that $Z$ is in $\mathscr{C}_{0}$.

Axiom [2, (2.4)]: here we suppose that we are given a system $X_{1} \stackrel{f_{1}}{\longrightarrow} X_{2} \stackrel{f_{2}}{\longrightarrow} \cdots$ in $\mathfrak{A}$, and use Definition 1.4 to get $X=\operatorname{hocolim} X_{i}$ and canonical maps $\underline{g_{i}}: X_{i} \longrightarrow X$.

Part (i): when $Z \in \mathscr{C}_{0}$, condition $\left(3^{\circ}\right)$ says that we can choose an X-preenvelope $Z \longrightarrow Y$ with $Y \in \mathscr{C}_{0}$. According to condition $\left(2^{\circ}\right)$, both functors $\operatorname{Hom}_{\mathfrak{I}}(Z,-)$ and $\operatorname{Hom}_{\mathfrak{I}}(Y,-)$ respect colimits of $\mathfrak{A}$-systems $A_{1} \longrightarrow A_{2} \longrightarrow \cdots$ where each arrow is an $\mathrm{X}$-monic. But then, Lemma 1.6 states that Brown's Axiom [2, (2.4.i)] is satisfied.

Part (ii): follows directly from Lemma 1.5.

Note again that the conditions listed in the statement of this theorem are frequently satisfied: When $\mathfrak{A}$ is abelian and closed under small colimits, conditions (A), (B) and (D) hold automatically. Condition (C) is not too outlandish. And conditions $\left(1^{\circ}\right)$ to $\left(4^{\circ}\right)$ are just finiteness-conditions on the objects of $\mathscr{C}_{0}$; they too will hold if we choose $\mathscr{C}_{0}$ to consist of sufficiently "finite" objects. An example could be finitely generated modules.

As mentioned, the point of introducing abstract homotopy-categories is that one can characterize the representable functors defined on them. [2, Thm. 2.8] states: suppose that $\overline{\mathscr{C}} 0$ functor $H: \mathscr{C} \longrightarrow$ Sets is representable precisely if $H$ is a homotopy-functor, i.e., precisely when $H$ satisfies the conditions [2, (2.6) and (2.7)].

Let us recall from [2] what " $\overline{\mathscr{C}_{0}}$ " and "a homotopy-functor" mean: 
The class $\overline{\mathscr{C}_{0}}$ consists of objects $Y$ for which the condition " $f: Y \longrightarrow Y^{\prime}$ is an isomorphism" is equivalent to the condition "for all $X \in \mathscr{C}_{0}$, the map $\operatorname{Hom}_{\mathscr{C}}(X, f)$ is bijective".

And as stated, a homotopy-functor is a functor satisfying [2, Cond. (2.6) and (2.7)]. Condition [2, (2.6)] says that $H$ should send coproducts to products. Condition [2, (2.7)] says that if we have one of the diagrams from Axiom [2, (2.3)],

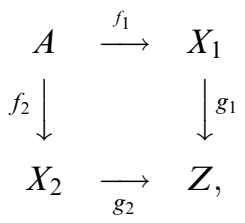

and $H\left(f_{1}\right)\left(u_{1}\right)=H\left(f_{2}\right)\left(u_{2}\right)$, then there exists $v \in H(Z)$ such that $H\left(g_{i}\right)(v)=u_{i}$. It is a nice point that in the case of the categories $\mathfrak{A}_{\mathrm{X}}$, condition [2, (2.7)] is equivalent to half-exactness of $H$ :

Lemma 2.2. Suppose that we are in the situation of Theorem 2.1, and let $H: \mathfrak{A}_{\mathbf{X}} \longrightarrow \mathbf{A b}$ be a contravariant additive functor. Let $U: \mathbf{A} \mathbf{b} \longrightarrow$ Sets be the forgetful functor.

Then $U \circ H$ satisfies [2, Cond. (2.7)] precisely when $H$ is half-exact in the sense of our Definition 1.8.

Proof. Suppose that $U \circ H$ satisfies [2, (2.7)], and let

$$
A \stackrel{a}{\longrightarrow} B \stackrel{b}{\longrightarrow} C \stackrel{c}{\longrightarrow} \Sigma(A)
$$

be a distinguished right-triangle. We need to see that $H(C) \stackrel{H(b)}{\longrightarrow}$ $H(B) \stackrel{H(a)}{\longrightarrow} H(A)$ is exact.

"Im $\subseteq$ Ker": this is true simply because $H$ is additive, since one can easily prove that $b \circ a=0$ (the usual argument from triangulated categories applies to prove that the composition of two consecutive arrows in a distinguished triangle is zero).

"Im $\supseteq$ Ker": by the proof of Theorem 2.1, part (2.3), the following is one of the diagrams from axiom [2, (2.3)]:

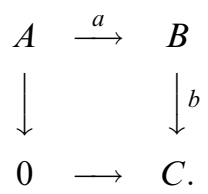

And if $u \in H(B)$ satisfies $H(a) u=0$, and we set $u_{1}=u$ and $u_{2}=0$, we have 
$H(a) u_{1}=H(0) u_{2}$, so since $H$ is a homotopy-functor, we can find $v \in H(C)$ such that $H(b) v=u_{1}=u$ (and $H(0) v=u_{2}=0$ ).

Suppose on the other hand that $H$ is half-exact, and let

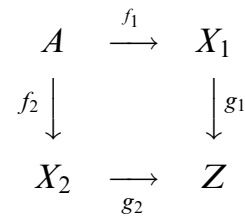

be one of the diagrams from Axiom [2, (2.3)]. Choose a distinguished righttriangle

$$
A \stackrel{\left(\begin{array}{c}
f_{1} \\
-f_{2}
\end{array}\right)}{\longrightarrow} X_{1} \coprod X_{2} \stackrel{\left(p_{1}, p_{2}\right)}{\longrightarrow} C \longrightarrow \Sigma(A) .
$$

We have $\left(p_{1}, p_{2}\right) \circ\left(\begin{array}{c}f_{1} \\ -f_{2}\end{array}\right)=0$, that is, $p_{1} f_{1}=p_{2} f_{2}$, so by the property characterizing $Z$ there exists $h: Z \longrightarrow C$ such that $h g_{i}=p_{i}$.

If we have $u_{i} \in H\left(X_{i}\right)$ with the property $H\left(f_{1}\right) u_{1}=H\left(f_{2}\right) u_{2}$, we can combine the $u_{i}$ to $\left(\begin{array}{l}u_{1} \\ u_{2}\end{array}\right) \in H\left(X_{1} \coprod X_{2}\right)$ and get

$$
H\left(\left(\begin{array}{c}
f_{1} \\
-f_{2}
\end{array}\right)\right)\left(\left(\begin{array}{l}
u_{1} \\
u_{2}
\end{array}\right)\right)=0
$$

and by the half-exactness of $H$, we can find $w \in H(C)$ such that $H\left(\left(p_{1}, p_{2}\right)\right) w=\left(\begin{array}{l}u_{1} \\ u_{2}\end{array}\right)$. But setting $v=H(h) w$, we see

$$
H\left(g_{i}\right) v=H\left(g_{i}\right) H(h) w=H\left(h g_{i}\right) w=H\left(p_{i}\right) w=u_{i},
$$

showing that $U \circ H$ satisfies [2, (2.7)].

\section{Application to derived categories}

In this short section, we shall apply the theory of section 2 to the derived category of a ring, recapturing in our Theorem 3.2 a special case of the Brown Representability Theorem proved as [11, Thm. 3.1] for abstract triangulated categories.

Let $A$ be a ring, and let $\mathfrak{A}$ be the additive category defined as follows: as objects, $\mathfrak{H}$ has all those $K$-projective complexes which consist of projective $A$-left-modules (see [13, Def. 1.1] for the definition of $K$-projectivity). As morphisms, $\mathfrak{A}$ has all chain-maps of complexes.

If we take $\mathfrak{U}$ modulo chain-homotopy, we get what we will call the classical homotopy-category of $\mathfrak{A}$. This category is isomorphic to the derived ca- 
tegory $D(A)$ of $A$-left-modules. This is a well-known consequence of the results of [13].

And in fact, we can obtain $\mathfrak{A}$ modulo chain-homotopy as the stable category $\mathfrak{A}_{\mathrm{X}}$ with respect to a suitable pre-enveloping class $\mathrm{X}$. To define $\mathrm{X}$, recall from [5, p. 28] that a chain-map $X \stackrel{f}{\longrightarrow} Y$ is called a semi-split injection if it is a split injection in each degree (the differentials do not play a role in this connection). A complex $I$ is called $S$-injective if, when we are given a semisplit injection $X \stackrel{f}{\longrightarrow} Y$ of arbitrary complexes, any chain-map $X \stackrel{g}{\longrightarrow} I$ extends to a chain-map $Y \stackrel{h}{\longrightarrow} I$ such that $h f=g$. Now let

$$
\mathrm{X}=\text { the } S \text { - injective } \mathfrak{A} \text {-complexes. }
$$

(Note that $S$-injectivity is defined by an extension-property in the category of all complexes, not just in $\mathfrak{A}$.) It is easy to prove, by imitating the arguments in $[5$, p. 28], that

- $\mathrm{X}$ is pre-enveloping in $\mathfrak{A}$; here one can use the same construction of preenvelopes as in [5, p. 28].

- The X-monics in $\mathfrak{A}$ are exactly the semi-split injections.

- A morphism $P \longrightarrow Q$ from $\mathfrak{A}$ is null-homotopic (in the normal definition from the theory of chain-homotopy) precisely when it factors through an object from $X$.

As a consequence of the last point, we see that as claimed, $\mathfrak{U}_{X}$ is isomorphic to the classical homotopy-category of $\mathfrak{A}$, that is, to $D(A)$.

In fact, as one can see by doctoring the arguments in [5, p. 28], we even get that the suspension-functor $\Sigma$ on $\mathfrak{A}_{\mathrm{X}}$ is equal to the usual shifting-functor on complexes, and that the notion of distinguished right-triangles in the righttriangulated category $\mathfrak{I}_{\mathrm{X}}$ coincides with the usual notion of distinguished triangles of complexes. Hence

$$
\left(\mathfrak{A}_{\mathrm{X}}, \Sigma, \Delta\right) \cong(D(A), \text { shift, the usual triangles }) .
$$

So the theory of derived categories over rings is contained in the theory of stable categories.

We can now prove

Proposition 3.1. Let $A$ be a ring. Let $\mathscr{C}=D(A)$ be the derived category, and let $\mathscr{C}_{0}$ be a full subcategory containing one element from each isomorphism-class which contains a bounded complex of finitely generated projectives (so $\mathscr{C}_{0}$ is small).

Then the pair $\left(\mathscr{C}, \mathscr{C}_{0}\right)$ is a homotopy-category in the sense of [2, p. 79]. Moreover, we have $\overline{\mathscr{C}_{0}}=\mathscr{C}$ (for the definition of $\overline{\mathscr{C}_{0}}$ see [2, p. 80], or above, after the proof of Theorem 2.1). 
Proof. As shown above, $\mathscr{C}=D(A)$ can be obtained as $\mathfrak{A}_{\mathrm{X}}$. So we shall apply Theorem 2.1 to this category. We must first consider conditions (A) to (D) on $\mathfrak{A}$ and $\mathrm{X}$.

Condition (A): if $\varphi: P \longrightarrow Q$ is an X-monic in $\mathfrak{A}$, we get a semi-split exact sequence of complexes,

$$
0 \rightarrow P \longrightarrow Q \longrightarrow R \rightarrow 0 \text {. }
$$

Since the sequence is semi-split, $R$ consists of projectives. And it is easy to see that $R$ is in fact $K$-projective, so $R$ is the cokernel in $\mathfrak{A}$ of the X-monic $\varphi$.

Condition (B): the existence of small coproducts in $\mathfrak{A}$ is clear.

Condition (C): if $\left\{P_{\alpha}\right\}$ is a small system from $\mathrm{X}$, every complex $P_{\alpha}$ is $S$ injective. So the coproduct $\coprod_{\alpha} P_{\alpha}$ is also $S$-injective, see [5, p. 28]. Therefore, the coproduct is in $\mathrm{X}$.

Condition (D): if we have a system $P_{1} \longrightarrow P_{2} \longrightarrow \cdots$ where all arrows are split injections, it will in fact be what [13, Def. 2.6(a)] calls an $\mathfrak{A}$-special direct system (strictly speaking, we need to add $P_{0}=0$ to the beginning of the system to satisfy [13]'s definition). We then see from [13, Cor. 2.8] that the system's colimit is $K$-projective. And it is clear that all modules in the colimit are projective (each module is the colimit of a sequence of split injections of projectives). So the colimit is in $\mathfrak{A}$.

Now look at conditions $\left(1^{\circ}\right)$ to $\left(4^{\circ}\right)$ from Theorem 2.1: it is easy to see that condition $\left(1^{\circ}\right)$ holds. Condition $\left(2^{\circ}\right)$ is well-known. Validity of condition $\left(3^{\circ}\right)$ follows from the concrete construction of pre-envelopes in $[5, \mathrm{p}$. 28]. And condition $\left(4^{\circ}\right)$ is easy to check.

So $\left(\mathscr{C}, \mathscr{C}_{0}\right)$ with $\mathscr{C}=\mathfrak{A}_{\mathrm{X}}$ is an abstract homotopy-category by Theorem 2.1 .

Lastly, we prove the proposition's statement about $\overline{\mathscr{C}_{0}}$. Since $\mathscr{C}$ is not just right-triangulated, but in fact triangulated, it is enough to see that if an object $Q \in \mathscr{C}$ has $\operatorname{Hom}_{\mathscr{C}}(P, Q)=0$ for all $P \in \mathscr{C}_{0}$, then $Q \cong 0$. But $\Sigma^{i}(A)$ (the ring itself placed in degree $-i)$ is in $\mathscr{C}_{0}$, and $\operatorname{Hom}_{\mathscr{C}}\left(\Sigma^{i}(A), Q\right)=h^{-i}(Q)$, and if this is always zero, $Q$ itself is isomorphic to zero (here we use that if a complex in $D(A)$ is exact, it is isomorphic to zero).

Theorem 3.2 (Cf. [11, Thm. 3.1]). Let $A$ be a ring. Let $H: D(A) \longrightarrow \mathbf{A b}$ be a contravariant additive functor.

Then the following two conditions are equivalent:

- (Representability): $H$ is representable in the sense that there exists an object $Y_{H} \in D(A)$ such that

$$
H(-) \cong \operatorname{Hom}_{D(A)}\left(-, Y_{H}\right) .
$$

- (Homology): $H$ is cohomological and sends small coproducts to the corresponding products. 
Proof. Assume that $H$ satisfies condition (Representability). It will then clearly send (small) coproducts to the corresponding products, and it is classical that $H$ is cohomological (the latter fact also follows from our Lemma 1.9). So $H$ satisfies condition (Homology).

Assume conversely that $H$ satisfies condition (Homology). Then the fact that $H$ is a cohomological functor implies that it is half-exact in the sense of Definition 1.8. For the moment, let us think of $H$ as a functor going into Sets. Then by Lemma 2.2, $H$ satisfies [2, Cond. (2.7)]. And since $H$ also satisfies [2, Cond. (2.6)], it is a homotopy-functor in Brown's sense.

So due to the facts stated in Proposition 3.1, if we regard $H$ as a functor to Sets, it is representable by [2, Thm. 2.8].

But by the Yoneda-lemma, [9, p. 61], when we have some natural transformation

$$
\varphi: \operatorname{Hom}_{D(A)}\left(-, Y_{H}\right) \longrightarrow H(-)
$$

of functors $D(A) \longrightarrow$ Sets, there exists an element $f \in H\left(Y_{H}\right)$ such that for any $X$ and $\xi$ we have $\varphi_{X}(\xi)=H(\xi)(f)$. In the case at hand, this prescription certainly makes each $\varphi_{X}$ a homomorphism of abelian groups.

So the established equivalence from $\operatorname{Hom}_{D(A)}\left(-, Y_{H}\right)$ to $H(-)$ is, in fact, a natural equivalence of functors $D(A) \longrightarrow \mathbf{A} \mathbf{b}$, and this proves condition (Representability).

\section{Application to Artinian rings}

This section applies the results of section 2 to the category of left-modules over a left-Artinian ring. It will be seen that, if we form the corresponding stable category with respect to the pre-enveloping class of injective modules, we get an abstract homotopy-category, and that in many cases, a good $\mathscr{C}_{0}$ can be found. Some consequences for functors defined on stable module-categories over Artinian rings are derived; the central one is expressed in Theorem 4.4.

We begin with a general observation: suppose that $\mathfrak{A}$ is an additive category, and let $\mathrm{X}$ be a class of objects of $\mathfrak{A}$ which is both pre-enveloping and pre-covering. Suppose that each $\mathrm{X}$-pre-envelope has a cokernel, and that each X-pre-cover has a kernel. By the general theory of [1], the stable category $\mathfrak{I}_{\mathrm{X}}$ is then equipped with a suspension-functor, $\Sigma$, and a loop-spacefunctor, $\Omega$. It is easy to prove that $(\Sigma, \Omega)$ are adjoint functors, a fact we need in this section. We omit the proof.

Now to the Artinian rings. Throughout this section, we let $R$ be a leftArtinian ring which has the property that if $M$ is a finitely generated leftmodule, then its injective envelope $E(M)$ is also finitely generated. By [8, 
Thm. 2], this is for instance the case if $R$ is PI, and so in particular if it is finite over its center.

We let $\mathfrak{A}$ stand for $\operatorname{Mod}(R)$, the category of $R$-left-modules, and let $\mathrm{X}$ stand for $\mathscr{I} n j(R)$, the class of all injective $R$-left-modules. Note that since $R$ is left-Artinian and therefore left-Noetherian, by [4, Prop. 2.2] the class $\mathscr{I} n j$ is pre-covering, so this places us in the situation described above: the suspension-functor $\Sigma: \mathfrak{A}_{\mathrm{X}} \longrightarrow \mathfrak{U}_{\mathrm{X}}$ has a right-adjoint functor $\Omega$. We write $\mathscr{C}=\mathfrak{A}_{\mathrm{X}}$, and let $\mathscr{C}_{0}$ be a full subcategory consisting of a representative from each isomorphism-class of finitely generated modules (so $\mathscr{C}_{0}$ is small).

Our basic result is the following:

Proposition 4.1. When $R$ is a ring as described in the introduction to this section, and we use the definitions given above, conditions $(A)$ to $(D)$ are satisfied by $\mathfrak{A}$ and $\mathrm{X}$, and conditions $\left(1^{\circ}\right)$ to $\left(4^{\circ}\right)$ from Theorem 2.1 are satisfied by $\mathscr{C}_{0}$.

So by Theorem 2.1, the pair $\left(\mathscr{C}, \mathscr{C}_{0}\right)$ is an abstract homotopy-category.

Proof. Conditions (A), (B) and (D) clearly hold. And condition (C) holds because $R$ is left-Artinian, and so in particular left-Noetherian.

Conditions $\left(1^{\circ}\right)$ and $\left(4^{\circ}\right)$ from Theorem 2.1 clearly hold. That condition $\left(2^{\circ}\right)$ holds is well-known. And condition $\left(3^{\circ}\right)$ is satisfied because we have required $R$ to be a ring such that if $M$ is finitely generated, then $E(M)$ is also finitely generated.

To be able to apply [2, Thm. 2.8], we need to see that $\overline{\mathscr{C}_{0}}=\mathscr{C}$. This is proved below in proposition $4.3\left(\overline{\mathscr{C}_{0}}\right.$ is defined in [2, p. 80], and above, after the proof of Theorem 2.1).

Lemma 4.2. Let A be a left-Noetherian ring, and consider the stable category

$$
\operatorname{Mod}(A)_{\mathscr{I} n j(A)} .
$$

If $C$ is a left-module with the property that for any finitely generated module $M$, we have

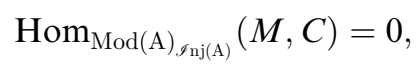

then $C$ is injective.

Proof. The assumption on $C$ says that any morphism $M \longrightarrow C$, with $M$ finitely generated, factors through $E(M)$. In particular, let $\mathfrak{a}$ be some leftideal of $A$, and let $a: \mathfrak{a} \longrightarrow C$ be a homomorphism. $\mathfrak{a}$ is finitely generated, so if $i: \mathfrak{a} \hookrightarrow E(\mathfrak{a})$ is the injection, we can find $e: E(\mathfrak{a}) \longrightarrow C$ such that $e i=a$. But if $j: \mathfrak{a} \hookrightarrow A$ is the canonical injection, we can also find $k: A \longrightarrow E(\mathfrak{a})$ 
such that $k j=i$, whence $a=e i=(e k) j$, showing that we have extended $a$ to $A$. By Baer's theorem, $C$ is injective.

Proposition 4.3. When $R$ is a ring as described in the introduction to this section, we have $\overline{\mathscr{C}_{0}}=\mathscr{C}$ in Browns's notation from $[2$, p. 80].

Proof. Let $\beta^{\prime}: B^{\prime} \longrightarrow B$ be a module-homomorphism with the property that when $M$ is finitely generated, $\pi\left(M, \beta^{\prime}\right): \pi\left(M, B^{\prime}\right) \longrightarrow \pi(M, B)$ is bijective (here we use Hilton's notation $\operatorname{Hom}_{\operatorname{Mod}(\mathrm{R})_{\mathscr{S}_{\mathrm{n}}(\mathrm{R})}}=\pi$ ). What we need to see is that $\beta^{\prime}$ is an isomorphism.

Use the dual of Lemma 1.2 to factor $\beta^{\prime}$ as $B^{\prime} \stackrel{\varphi}{\longrightarrow} A \stackrel{\alpha}{\longrightarrow} B$, where $\varphi$ is an $\mathrm{X}$-homotopy-equivalence, and $\alpha$ an X-epic. Then $\pi\left(M, \beta^{\prime}\right)$ is bijective precisely when $\pi(M, \underline{\alpha})$ is bijective. By the assumption on $\beta^{\prime}$, we thus know that $\pi(M, \underline{\alpha})$ is bijective for any finitely generated $M$, and need to prove that $\underline{\alpha}$ is an isomorphism.

So let us look at $\alpha: A \longrightarrow B$. By construction, it is $\mathrm{X}$-epic. In fact, it is also a surjective homomorphism: let $b$ be an element of $B$. Let $i: R b \hookrightarrow B$ be the canonical inclusion. Since $R b$ is finitely generated, our assumption tells us that in the homotopy-category, we can lift $\underline{i}$ through $\underline{\alpha}$, that is, we can find a homomorphism $\rho: R b \longrightarrow A$ such that $\underline{\alpha} \circ \underline{\rho}=\underline{i}$. But by the dual of Lemma 1.1, there will then exist a homomorphism $\tilde{\rho}: R b \longrightarrow A$ such that $\alpha \circ \tilde{\rho}=i$, so $b$ is seen to be in the image of $\alpha$, proving its surjectivity.

Now construct a distinguished left-triangle $\Omega(B) \longrightarrow C \longrightarrow A \stackrel{\alpha}{\longrightarrow} B$. For any module $M$, we obtain a long-exact sequence from the dual of Lemma 1.9,

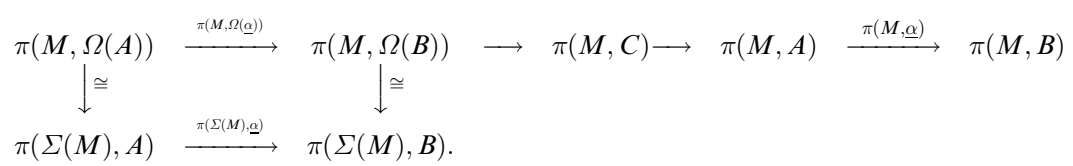

The vertical arrows are adjunction-isomorphisms. If $M$ is finitely generated, we can suppose that $\Sigma(M)$ is finitely generated (since $E(M)$ is), and then by assumption, both maps $\pi(M, \underline{\alpha})$ and $\pi(\Sigma(M), \underline{\alpha})$ are isomorphisms. Since $\pi(\Sigma(M), \underline{\alpha})$ is an isomorphism, so is $\pi(M, \Omega(\underline{\alpha}))$. But when both $\pi(M, \Omega(\underline{\alpha}))$ and $\pi(M, \underline{\alpha})$ are isomorphisms, the diagram shows that we must have $\pi(M, C)=0$. And when this holds for all finitely generated $M$ 's, we know from Lemma 4.2 that $C$ is an injective module.

However, $\alpha$ is an X-epic, so by the dual of [1, Prop. 2.10], the module $C$ can be taken to be $\operatorname{Ker}(\alpha)$. Collecting everything, we see that $\alpha$ is a surjective homomorphism with kernel equal to an injective module - that is, $\alpha$ splits.

Of course, this shows that $\pi(M, \underline{\alpha})$ is surjective for any $M$. And the fact that $C \cong 0$ in $\operatorname{Mod}(R)_{\mathscr{I}_{n j}(R)}$ along with the exact sequence $(* *)$ shows that $\pi(M, \underline{\alpha})$ is injective for any $M$. So $\underline{\alpha}$ is an isomorphism, and this is what we want. 
This places us in the best possible position, from which the central result can be obtained:

THEOREM 4.4. Let $R$ be a ring as described in the introduction to this section, and let $H: \operatorname{Mod}(R)_{\mathscr{I} n j(R)} \longrightarrow \mathbf{A b}$ be a contravariant additive functor.

Then the following two conditions are equivalent:

- (Representability): $H$ is representable in the sense that there exists a module $Y_{H}$ such that

$$
H(-) \cong \pi\left(-, Y_{H}\right)
$$

- (Homology): $H$ is half-exact and sends small coproducts to the corresponding products.

Proof. If $H$ satisfies condition (Representability), it clearly satisfies condition (Homology) (use Lemma 1.9).

On the other hand, if $H$ satisfies condition (Homology), we know from Lemma 2.2 that when we regard $H$ as a functor to Sets, it is a homotopyfunctor in the sense of [2, p. 80]. So condition (Representability) for $H$ as a functor into Sets follows immediately when we feed Propositions 4.1 and 4.3 into [2, Thm. 2.8].

To get the equivalence of functors into $\mathbf{A b}$, we use the same standardtrick with Yoneda's lemma as in the proof of Theorem 3.2.

Let us show a few applications of the theory:

ExAmple 4.5. Let $R$ be a ring as described in the introduction to this section, and let

$$
F: \operatorname{Mod}(R) \longrightarrow \mathbf{A b}
$$

be a contravariant additive functor which sends small coproducts to the corresponding products. Then for each $i \geq 1$, there exists an $R$-module $A_{i}$ such that

$$
L_{i} F(-) \cong \pi\left(Q(-), A_{i}\right) .
$$

By $Q$, we mean the canonical functor $Q: \operatorname{Mod}(R) \longrightarrow \operatorname{Mod}(R)_{\mathscr{I} n j(R)}$.

Proof. When $i \geq 1$, the derived functor $L_{i} F(-)$ vanishes on injectives, and so factors through $\operatorname{Mod}(R)_{\mathscr{I} n j(R)}$. It is thus enough to represent it when we view it as defined on $\operatorname{Mod}(R)_{\mathscr{I} n j(R)}$.

$L_{i} F(-)$ is half-exact in the sense of Definition 1.8; this follows from the fact that $L_{i} F(-)$ is half-exact in the usual sense, and from [1, Prop. 2.10] which states that any distinguished right-triangle in $\operatorname{Mod}(R)_{\mathscr{I} n j(R)}$ comes from a short-exact sequence in $\operatorname{Mod}(R)$.

And each $L_{i} F(-)$ sends small coproducts to the corresponding products: this follows because a small coproduct of injective resolutions is again an 
injective resolution, which again follows because a small coproduct of injectives is injective over the left-Artinian and hence left-Noetherian ring $R$.

Now use Theorem 4.4.

Next let us be somewhat more systematic. Since we think of the stable category $\operatorname{Mod}(R)_{\mathscr{I} n j(R)}$, and more generally of abstract right-triangulated categories, as analogous to the homotopy-category from algebraic topology, it is very obvious to make the two following definitions:

Definition 4.6. (For abstract right-triangulated categories; compare [6, p. 8]). Let $(\mathfrak{C}, \Sigma, \Delta)$ be a right-triangulated category. A cohomology-theory on $(\mathfrak{C}, \Sigma, \Delta)$ is a collection

$$
\left(H^{i}, \delta^{i}: H^{i} \stackrel{\cong}{\longrightarrow} H^{i+1} \circ \Sigma\right)_{i \in \mathrm{Z}},
$$

where each $H^{i}$ is a contravariant half-exact functor from $\mathbb{C}$ to $\mathbf{A b}$, and each $\delta^{i}$ is a natural equivalence.

Definition 4.7. (Cf. [6, p. 14]). Let $A$ be a left-Noetherian ring. An $\Omega$ spectrum in $\operatorname{Mod}(A)_{\mathscr{I} n j(A)}$ is a collection

$$
\left(B^{i}, \underline{b^{i}}: B^{i} \stackrel{\cong}{\longrightarrow} \Omega\left(B^{i+1}\right)\right)_{i \in \mathrm{Z}},
$$

where each $B^{i}$ is an $A$-module, and each $\underline{b^{i}}$ is an isomorphism in $\operatorname{Mod}(A)_{\mathscr{I} n j(A)}$.

Note that using stable homotopy, we can easily construct cohomology-theories in the sense of Definition 4.6. It was pointed out to me by Alex Martsinkovsky that theories obtained in this way are isomorphic to the so-called Vogel cohomology-theories of modules treated in [10], and thus have a number of useful properties.

We can now prove that the connection between cohomology-theories and $\Omega$-spectra is as expected from the topological theory, cf. [3, Thms. II and III]:

Proposition 4.8. Let $R$ be a ring as described in the introduction to this section, and let $H=\left(H^{i}, \delta^{i}: H^{i} \stackrel{\cong}{\longrightarrow} H^{i+1} \circ \Sigma\right)_{i \in \mathrm{Z}}$ be a cohomology-theory defined on $\operatorname{Mod}(R)_{\mathscr{I} n j(R)}$. Suppose that each $H^{i}$ sends small coproducts to the corresponding products.

Then there exists an $\Omega$-spectrum $B=\left(B^{i}, \underline{b^{i}}: B^{i} \stackrel{\cong}{\longrightarrow} \Omega\left(B^{i+1}\right)\right)_{i \in \mathrm{Z}}$ such that

$$
H^{i}(-) \cong \pi\left(-, B^{i}\right)
$$

for each $i$, and such that the equivalences $\delta^{i}$ are the compositions 


$$
\pi\left(-, B^{i}\right) \stackrel{\pi\left(-, \underline{b^{i}}\right)}{\longrightarrow} \pi\left(-, \Omega\left(B^{i+1}\right)\right) \longrightarrow \pi\left(\Sigma(-), B^{i+1}\right)
$$

(the last arrow is the adjunction-isomorphism). The $\Omega$-spectrum $B$ is unique in an obvious sense.

Conversely, given an $\Omega$-spectrum $B$, we can use the two displayed formulae to define a cohomology-theory $H$, in which each $H^{i}$ will send small coproducts to the corresponding products.

Proof. The last statements, about uniqueness of the spectrum $B$, and the possibility of using a $B$ to define an $H$, are clear. So we just need to see that we can obtain $B$ when we are given the cohomology-theory $H$.

However, by Theorem 4.4, we can certainly find modules $B^{i}$ such that $H^{i}(-) \cong \pi\left(-, B^{i}\right)$. The $\delta^{i}$ 's then give equivalences of functors

$$
\pi\left(-, B^{i}\right) \cong \pi\left(\Sigma(-), B^{i+1}\right)
$$

and using the adjunction, we can write this isomorphism as $\pi\left(-, B^{i}\right) \cong \pi\left(-, \Omega\left(B^{i+1}\right)\right)$. But now we just apply Yoneda's lemma to get the isomorphisms $\underline{b^{i}}$.

ACKNOWLedgement. I would like to thank professor Alex Martsinkovsky for a number of very illuminating discussions of Hilton's homotopy-theory for modules, and for bringing [1] and [7] to my attention. I have also had benefit from several discussions with Jesper Grodal.

I thank professor Søren Jøndrup for pointing out the reference [8] to me.

Part of this work was carried out at the Institut Mittag-Leffler in Stockholm. I thank the Institute for its hospitality, and Anders Thorup for arranging my stay.

\section{REFERENCES}

1. A. Beligiannis and N. Marmaridis, Left triangulated categories arising from contravariantly finite subcategories, Comm. Algebra 22 (1994), 5021-5036.

2. E. H. Brown, Abstract homotopy theory, Trans. Amer. Math. Soc. 119 (1965), 79-85.

3. E. H. Brown, Cohomology theories, Ann. of Math. (2) 75 (1962), 467-484.

4. E. E. Enochs, Injective and flat covers, envelopes and resolvents, Israel J. Math. 39 (1981), 189-209.

5. D. Happel, Triangulated Categories in the Representation Theory of Finite Dimensional Algebras, London Math. Soc. Lecture Note Ser. 119, Cambridge University Press, Cambridge, 1988.

6. P. J. Hilton, General Cohomology Theory and K-theory, London Math. Soc. Lecture Note Ser. $1,1971$.

7. P. J. Hilton, Homotopy Theory and Duality, Gordon and Breach, New York, 1965.

8. A. V. Jategaonkar, Certain injectives are artinian, in "Noncommutative Ring Theory", Lecture Notes in Math. 545 (1976), 128-139. 
9. S. MacLane, Categories for the Working Mathematician, Graduate Texts in Math. 5, 1971.

10. A. Martsinkovsky, New homological invariants for modules over local rings, I, J. Pure Appl. Algebra 110 (1996), 1-8.

11. A. Neeman, The Grothendieck duality theorem via Bousfield's techniques and Brown Representability, J. Amer. Math. Soc. 9 (1996), 205-236.

12. D. G. Quillen, Homotopical Algebra, Lecture Notes in Math. 43 (1967).

13. N. Spaltenstein, Resolutions of unbounded complexes, Compositio Math. 65 (1988), 121-154.

MATEMATISK AFDELING

KøBENHAVNS UNIVERSITET

UNIVERSITETSPARKEN 5

DK-2100 KøBENHAVN Ø

DENMARK

Email address: popjoerg@math.ku.dk,www.math.ku.dk/ popjoerg 